\title{
THE DEVELOPMENT OF A NOVEL MINIATURISED MODULAR PLATFORM FOR WIRELESS SENSOR NETWORKS
}

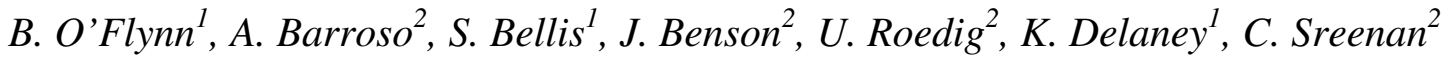 \\ ${ }^{1} \mathrm{NMRC}-\{$ brendan.oflynnl sbellisl kdelaney $\} @$ nmrc.ie \\ ${ }^{2}$ Mobile \& Internet Systems Laboratory, University College Cork, Ireland - \\ \{a.barrosolj.bensonlu.roediglc.sreenan\}@cs.ucc.ie
}

\begin{abstract}
Wireless sensor networks are collections of autonomous devices with computational, sensing and wireless communication capabilities. Research in these networks has been growing steadily in the past few years given the wide range of applications that can benefit from such a technology.

In this paper, the development of a highly modular and miniaturized wireless platform for sensor networks is described. The system incorporates a radio transceiver (operating in the $2.4 \mathrm{GHz}$ ISM Band) with embedded protocol software to minimize power consumption and maximize data throughput. Additional input capability for sensor and actuator integration can be incorporated seamlessly due to the modular nature of the system. The total system is packaged in a modular $25 \mathrm{~mm}$ cubed form factor.
\end{abstract}

\section{INTRODUCTION}

Several applications, such as precision farming, military field monitoring and seismic activity monitoring require long lived deployments of possibly a very large number of wireless sensors. As hardware becomes cheaper and smaller, more of these applications are likely to appear. Given the wide range of applications, flexibility is a desirable characteristic for a wireless sensor network platform. Furthermore, the design must aim powerefficiency for untethered, long-lived operation. Miniaturization is also important for deeply embedded applications where space is constrained. This paper describes a highly modular, miniaturized wireless sensor platform that addresses the issues of flexibility, powerefficiency and size (Figure 1).

The platform was developed as part of the D-Systems project [1] at University College Cork. On the hardware side, the key objective of the project is to produce miniaturized autonomous sensing units that can be easily

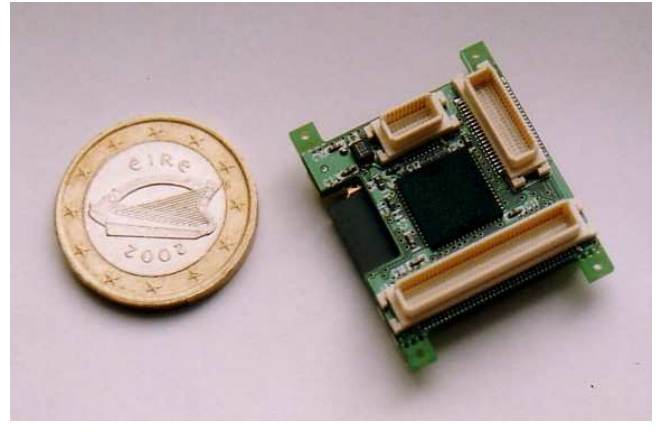

Figure 1 - RF Transceiver Layer

deployed and maintained in the everyday environment. The target sensing module is a $5 \mathrm{~mm}$ cube incorporating commercial-off-the-shelf (COTS) microsensors, ICs for signal processing, computation, and wireless communications, and a power source combined together within a highly innovative microelectronics packaging configuration. On the software side, the contribution will consist of a power aware operating system for the miniaturized autonomous sensing units that leverages the unique capabilities provided by the hardware.

The rest of this paper is organized as follows: In Section 1, a detailed description of the miniaturized modular platform is presented with emphasis on the RF transceiver layer; Section 2 provides an overview of the software architecture used and discusses the requirements for power efficient operation of the radio transceiver; Section 3 introduces the design of a protocol addressing the requirements of power-efficient communication between modules; Section 4 presents details on the implementation of the protocol; Finally, section 5 outlines application projects employing the described platform.

\section{MINIATURIZED MODULAR PLATFORM}

\subsection{Platform Overview}

The hardware platform is a Lego-like $25 \mathrm{~mm} \times 25 \mathrm{~mm}$ stackable system. Its modular nature lends itself to the 


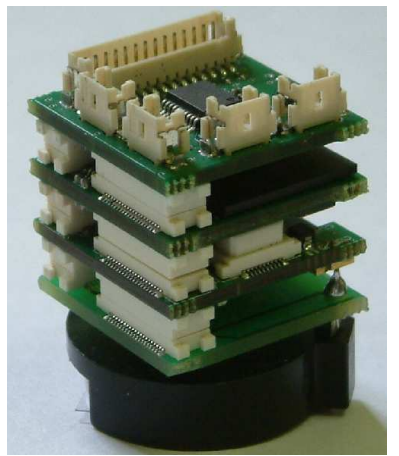

Figure 2 - Stack of Layers

development of numerous layers for use in different application scenarios. Layers can be combined in an innovative plug and play fashion and include communication, processing, sensing and power. The communication layer is comprised of a microcontroller, RF transceiver and embedded antenna. An FPGA for highspeed DSP forms the processing layer while various application specific sensors, as well as a generic sensor interface/communications layer have been developed for the sensing layer. The power layer may include batteries or other energy supply mechanisms such as solar cells.

The stackable configuration (Figure 2) enables ease of connectivity between layers depending on the system level requirements.

Modules use a stackable connector system to make the electrical and mechanical interconnections between layers. These high-density connectors have $0.5 \mathrm{~mm}$ pitch and are available in a range of interlayer spacing from $5 \mathrm{~mm}$ to $8 \mathrm{~mm}$ to allow for different component heights on the PCBs. The connectors facilitate an 80 pin general purpose bus and a 40 pin bus for configuration and data transfer between modules.

The RF transceiver layer also has a 20 pin connector allowing four low noise analog input channels for integrating sensitive analog sensors directly to the microcontroller part of this layer.

Due to its modularity, the platform has proved to be an invaluable developmental tool in the evaluation of power constrained networking protocols and applications in mobile wireless sensor systems.

\subsection{RF Transceiver Layer}

The transceiver/microcontroller layer was developed to provide RF communications capability between sensor nodes. The layer incorporates a microcontroller driving a transceiver operating in the $2.4 \mathrm{GHz}$ ISM band. It can be used "stand alone" with the processing power in the microcontroller driving system control, communication protocols and data processing, or can connect with the
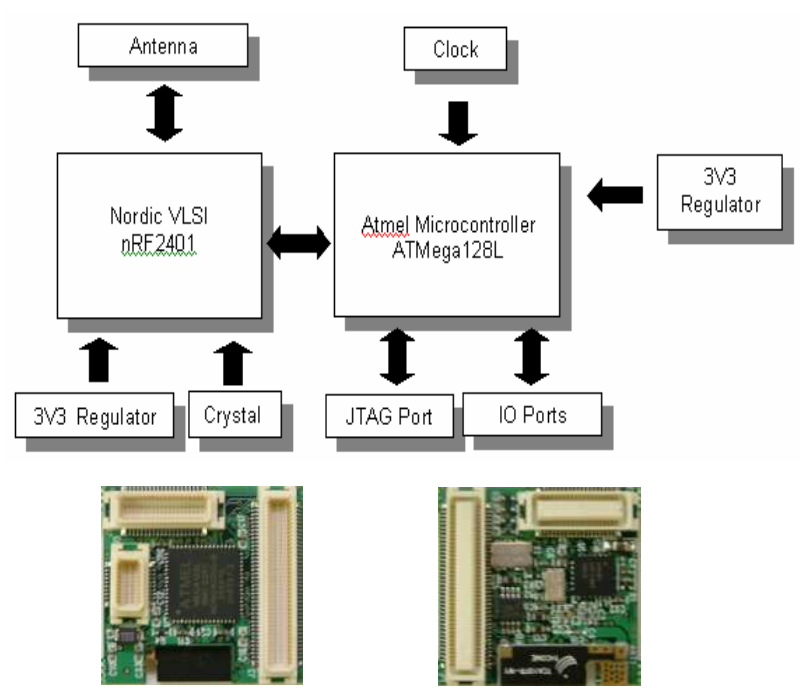

Figure 3 - Block Diagram and final
implementation of RF transceiver layer

separate battery module, FPGA and sensor layers depending on the mobility/portability requirements of the end user. Figure 3 shows the top and bottom views of the transceiver system layer along with a block diagram showing the main components on the module.

The transceiver (the nRF2401[2] from Nordic VLSI) consists of a fully integrated frequency synthesizer, a power amplifier, a crystal oscillator and a modulator. Output power and frequency channels are easily programmable. Since current consumption is required to be as low as possible, a built-in power down mode makes energy saving easily realisable. The system layer also features an on board $50 \Omega$ antenna. The embedded microcontroller is the ATMega128L[3], an 8-bit microcontroller with 128 Kbytes in-system programmable flash, allowing the user to program the device with custom communication protocols.

As the system is envisaged to operate in mobile sensor applications, low power consumption is essential. Therefore, power efficiency considerations were taken into account from the beginning of the design phase. The transceiver is able to operate in shockburst mode, which uses on-chip buffers to clock in data at a low data rate and transmit it at a very high rate. This feature greatly reduces power consumption. Furthermore, putting several high speed communication signal processing into the nRF2401 reduces current consumption, lowers system cost (by facilitating the use of a less expensive microcontroller), and greatly dilutes the risk of 'on-air' collisions due to short (high speed) transmission time. Low power consumption requirements are also assisted by the Atmel microcontroller through its power-down modes. 


\section{COMMUNICATION CONTROL USING TINYOS}

\subsection{TinyOS Overview}

The platform runs a tailored version of TinyOS[4], an operating system designed at UC Berkeley and engineered to run in hardware platforms with severe resource constraints. The entire system can be described in terms of a graph of components and a scheduler. Components encapsulate functionality and state and provide well defined, bi-directional interfaces that can be "wired" to other components forming complex applications. The scheduler provides deferred execution of non-time critical and computationally intensive sequences of operations.

In TinyOS, "active messages" [5] is a way to structure the communication stack in sensor devices. According to this paradigm, every active message includes an application-level handler to be invoked on the destination node upon arrival. This communication stack is encapsulated in the GenericComm component of TinyOS 1.1.

\subsection{Energy-efficient radio operation}

The radio module of a wireless sensor platform is a major source of energy consumption. Given that many such platforms are powered by energy constrained batteries, it is essential to operate the radio in an efficient way to attain acceptable lifetime figures. Energy efficient operation of radios is achieved essentially by keeping them in power-saving mode or "sleeping" as often as possible. Sleeping states generally consume orders of magnitude less energy than active states while presenting good transition time delays when activation is required. The nRF2401 radio used in the platform consumes about 1000 times less energy in sleep mode than when in active mode (transmitting/receiving/listening). The transition time from sleep to active mode takes at most $200 \mu$ s, far less than the $3 \mathrm{~ms}$ required to transition from power-down to active state.

\subsection{Radio Control Requirements}

A simple approach to achieve energy-efficient radio operation is exemplified by the active message communication stack for the Mica[6] sensor platform. The stack provides energy-efficient operation of the Chipcon CC1000 radio by turning it periodically on and off. Since communication can only take place when the radio is on, a transmitter is required to send a packet with a longer preamble in order to guarantee it will be seen by the receiver. Once a receiver detects a packet, it keeps its radio on and receives at the full data rate [7].

As the transceiver in the platform was selected for its capability to provide low power shockburst

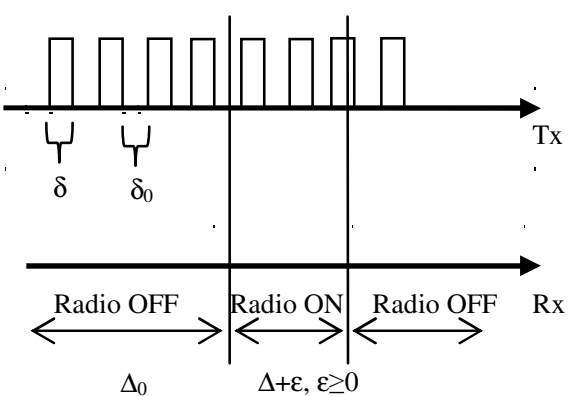

Figure 4 - Energy-Efficient Operation of Radio

communication capability in the $2.4 \mathrm{GHz}$ ISM band, this technique cannot be adopted directly, as its radio when operating in this fashion imposes a fixed size to the packets transmitted. Since the length of packets cannot be increased to compensate for the periodic unavailability of receivers, the energy-efficient mechanism has to be adapted. The design of a protocol addressing the requirement of fixed size packets in low power applications is described next.

\section{RADIO CONTROL DESIGN}

\subsection{Description}

While listening for incoming packets, a node periodically switches on and off its radio in order to reduce the energy consumption when packets are not being transmitted. The radio duty cycle period is represented as $P=\Delta+\Delta_{0}$, where $\Delta$ is the time the radio remains active and $\Delta_{0}$ is the time the radio rests in sleeping mode. The duty cycle ratio, or duty cycle for short, is defined as:

$$
\text { Duty Cycle }=\Delta P=\Delta\left(\Delta+\Delta_{0}\right)
$$

A sender, on the other hand, ensures its packet will be received at the destination by transmitting it multiple times as depicted in Figure 4.

Individual packets are acknowledged and retransmission ceases with the reception of the first ACK.

\subsection{Parameters}

The trail of packets is defined by three parameters:

- Number of retransmissions $(n)$

- Time between packets $\left(\delta_{0}\right)$

- Packet transmission time $(\delta)$

In order to ensure at least one packet of a trail will always be received at the destination, a proper relation between these parameters and $\Delta, \Delta_{0}$ must be observed. First, the active portion of duty cycle $\Delta$ must be such that: 
Furthermore, to assure overlapping between transmission and listening activities, the number of retransmissions $n$ needs to comply with the following inequality:

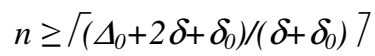

In general, the values of $\delta$ and $\delta_{0}$ should be as small as possible, as they influence, according to (2), the minimal possible active time $\Delta$ of the duty cycle. The duration of the time $\Delta$ determines message delay and throughput.

\subsection{Back-Off Mechanism}

Transmissions can take place at any time and therefore the medium access control protocol (MAC) proposed is contention-based. In order to reduce the probability of collisions between nodes willing to access the medium at the same time, a back-off mechanism is needed. Before a node starts transmitting a packet, it must listen to the medium for a period equal to $\Delta$. If no transmission is in progress, the node can begin its own transmission. Otherwise, a random delay is imposed before the procedure described initiates again.

\section{RADIO CONTROL IMPLEMENTATION}

The platform uses the nRF2401 single chip $2.4 \mathrm{GHz}$ transceiver from Nordic[2]. The main characteristics of this radio are GFSK modulation, data rates from 0 to $1 \mathrm{Mbps}$, frequency range from 2400 to $2524 \mathrm{MHz}$ and multi-channel operation. The transceiver can operate in active (RX/TX) or stand-by ("sleeping") mode. While active, the energy consumption is around $18 \mathrm{~mA}$. Sleeping, the consumption is reduced to $12 \mu \mathrm{A}$.

The transceiver has two active modes: direct and shockburst. In direct mode, the radio is essentially fully controlled from external hardware while in shockburst several tasks are pushed into the radio hardware.

The shockburst mode uses on-chip FIFO to clock in data at possibly low data rates and transmit at a very high rate. By transmitting faster, the radio can quickly return to a power saving mode and therefore energy consumption is reduced. This principle is depicted in figure 5. The data transfer speed can be set to $250 \mathrm{kbps}$ or $1 \mathrm{Mbps}$.

The radio packet format of the transceiver, when in shockburst mode, is shown in figure 7 . The packet has a fixed size and the transceiver automatically adds the preamble and CRC fields releasing the microcontroller of these tasks.

When a packet is received, the radio checks the address and CRC fields. If the packet is addressed to a different

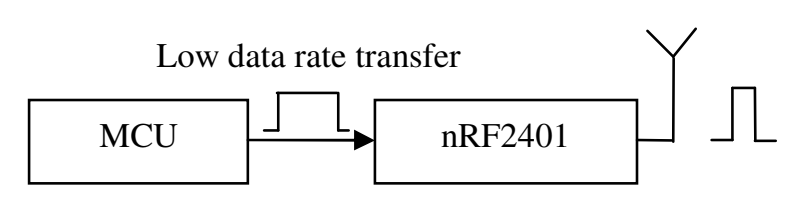

Figure 5 - Shockburst mode operation of the nRF2401 Radio

\begin{tabular}{|} 
Preamble Address & \multicolumn{1}{c}{ Payload } & \multicolumn{1}{c}{ CRC } \\
\hline 4 or 8 & $8-40$ & & $8-16$ \\
\hline
\end{tabular}

Figure 6 - Radio packet format in shockburst mode

radio unit or is corrupted then it is dropped. Only undamaged packets addressed to the unit generate interruptions to the microcontroller.

\subsection{Impact on Active Message Libraries}

The characteristics of the nRF2401 Nordic radio used in the platform have two immediate impacts on the implementation of active messages. First, unless a fragmentation layer is introduced, the active message must fit in the payload field shown in Figure 6. In the current tailored implementation of TinyOS, the radio address field uses 16 bits and the CRC field occupies 16 other bits. Therefore, only 224 bits are available for an active message. In other words, the maximum data payload for the active message packet is 21 bytes. The protocol implementation therefore fragments/reassembles active messages as needed.

A second impact of the radio characteristics on the implementation of active messages for the platform derives from the presence of redundant fields in the radio and active message packets. The nRF2401 radio handles CRC automatically in shockburst and therefore the embedded TinyOS control software is liberated from this task.

The radio physical address could also be set to match the active message address. This way the software would also be freed from processing packets addressed to other nodes. However, this approach is not recommended for the nRF2401 radio since it prevents monitoring channel activity for collision avoidance. The only way to monitor 
channel activity in this radio is to select a common radio address to all nodes. This way, the radio will be able to generate interruptions to the microcontroller when any packet is transmitted within the node's communication range. All packets are captured and the ones with different destinations are discarded by the software at a later stage.

\subsection{Implementation Parameters}

Sender transmitting time $(\delta)$

The nRF2401 radio is set to operate in shockburst mode at $250 \mathrm{kbps}$. Each packet transmitted has a fixed size of 264 bits (preamble of 8 bits). Therefore the transmitting time $\delta$ of a packet is approximately $1.1 \mathrm{~ms}$.

\section{Interval between retransmissions $\left(\delta_{0}\right)$}

The interval between retransmissions of packet replicas is a function of the hardware speed and the code controlling the radio operation. Guaranteeing a fixed value for this interval requires inhibiting hardware interruptions in the microcontroller for long periods of time, compromising system responsiveness.

In the implementation of the protocol, interruptions are not inhibited during data transfer from the microcontroller to the radio. Therefore a fixed value for $\delta_{0}$ or even an upper bound for this interval cannot be enforced. However, if a lower bound for $\delta_{0}$ is known, enough overhead can be added to the receiver's listening time $\Delta$ to ensure with high probability that inequality (2) is observed. As long as the aggregated amount of time spent with interruptions during the transmissions of two consecutive replicas does not cause the violation of the referred inequality, communication will be successful through retransmissions. For the protocol implementation, the minimum value of $\delta_{0}$ was empirically identified to be $9 \mathrm{~ms}$.

\section{Receiver listening time $(\Delta)$}

The listening time was selected to provide enough slack to accommodate jitter in the time between retransmissions. The maximum jitter assumed during retransmissions is $3 \mathrm{~ms}$. If the handling of interruptions is kept brief, then this value can accommodate many concurrent events. Applying $\delta_{0}=9 \mathrm{~ms}+3 \mathrm{~ms}$ and $\delta=1.1 \mathrm{~ms}$ to inequality (2) implies a value for $\Delta$ greater than or equal to $14.2 \mathrm{~ms}$.

Receiver sleep time $\left(\Delta_{0}\right)$

The receiver sleep time was selected to fix the radio duty cycle at $10 \%$. Applying $\Delta=15 \mathrm{~ms}$ to expression (1) and solving for $\Delta_{0}$ results in a value equal to $135 \mathrm{~ms}$.

Number of retransmissions ( $n$ )

According to expression (3), $\delta=1.1 \mathrm{~ms}, \delta_{0}=9 \mathrm{~ms}, \Delta$ $=15 \mathrm{~ms}$ and $\Delta_{0}=135 \mathrm{~ms}$ imply $\mathrm{n} \geq 15$.

\section{EXAMPLE APPLICATION AREAS}

The described platform has been used in several projects in applications ranging from intelligent environments to agriculture. The following paragraphs outline some of these projects.

\section{"E"'graculture Sensor Application}

Agriculture is an especially suitable application area for the developed platform given the difficulty in wiring cultivated areas. In this context, a thermistor layer for agricultural use was developed. The circuit was designed to operate using minimal current (microamps) and to activate an alarm on over-temperature. This alarm is connected to the RF transceiver layer on a interrupt pin enabling the system to operate in low power consumption "sleep mode" until an over-temperature alarm is sensed. The transceiver then transmits to actuators that trigger irrigation. A solar power layer was also developed for energy harvesting.

\section{E-Objects}

As part of the eGadgets[8] Project funded by the European Community under the "Information SocietyTechnologies" Programme, intelligence has been integrated into every day objects, to interact with users unobtrusively and develop ambient systems.

\section{Parking/Traffic Management}

In cooperation with the Cork City Council, a system for parking and traffic management is being prototyped..

\section{CONCLUSIONS AND FUTURE WORK}

This paper describes a highly modular, miniaturized wireless sensor platform that addresses the issues of flexibility, power-efficiency and size. The modular nature of the system, and the plug and play capability of the stacking system developed has proved to be an invaluable developmental tool in the evaluation of power constrained networking protocols, ambient system networks, transducer networks and mobile sensor based systems.

Future work in this area, already underway, is the implementation of a transceiver in the $433 \mathrm{MHz}$ band and a ZigBee[9] compliant transceiver layer. The development of a $10 \mathrm{~mm}$ and a $5 \mathrm{~mm}$ platform is part of the ongoing work.

\section{ACKNOWLEDGMENTS}

Thanks are due to Enterprise Irelands Informatics Initiative for funding this project, The NMRC and University College Cork, for providing the facilities to carry out the work and all the Microelectronics 
Applications Integration team in NMRC and the Mobile \& Internet Systems team in University College Cork for their support in developing this system.

\section{REFERENCES}

[1] The D-Systems Project [online], http://www.cs.ucc.ie/misl/ dsystems

[2] nRF2401 Single Chip 2.4GHz Radio Transceiver Data Sheet [online], http://www.nvlsi.no/

[3] ATMEL Corporation [online], http://www.atmel.com

[4] TinyOS Community Forum [online], http://www.tinyos.net

[5] J. Hill, P. Bounadonna and D Culler. "Active Message Communication for Tiny Network Sensors." [online] http://webs.cs.berkeley.edu/tos/media.html

[6] Crossbow Technology Inc. [online], http://www.xbow.com

[7] P. Levis, S. Madden, D. Gay, J. Polastre, R. Szewczyk, A. Woo, E. Brewer and D. Culler. "The Emergence of Networking Abstractions and Techniques in TinyOS." Proc of the 1st USENIX/ACM Symposium on Networked Systems Design and Implementation (NSDI2004), pp. 1-14, 2004

[8] e-Gadgets [online], http://www.extrovert-gadgets.net/

[9] Zigbee Allience [online], http://www.zigbee.org 